\title{
O GÊNERO RHIPSALIS GÄRTNER (CACTACEAE), NO ESTADO DE SÃO PAULO. II. ESPÉCIES COM RAMOS APLANADOS ${ }^{1}$
}

\author{
Julio Antonio Lombardi ${ }^{2}$
}

Recebido em 27.10.92. Aceito em 29.05.95.

\begin{abstract}
RESUMO: (O gênero Rhipsalis Gärtner (Cactaceae), no Estado de São Paulo. II. Espécies com ramos aplanados). Rhipsalis Gärtner é um gênero de Cactaceae de hábito epifítico ou rupícola, de ampla distribuição na América do Sul e Central, atingindo inclusive a África tropical e ilhas do Oceano Índico. Este trabalho conclui um estudo que caracterizou morfológicamente as espécies do gênero Rhipsalis que ocorrem no Estado de São Paulo, possibilitando o reconhecimento das espécies e a construção de uma chave de identificação. Entre as espécies do gênero que possuem artículos predominantemente aplanados foram reconhecidas seis espécies ocorrentes no Estado.
\end{abstract}

Palavras chave: Rhipsalis, taxonômia, ramos aplanados

\begin{abstract}
The genus Rhipsalis Gärtner (Cactaceae), in São Paulo State. II. Species with flatened joints). Rhipsalis Gärtner is a genus of epiphytic or rupicolous Cactaceae, with a wide distribution in South and Central America. It also occurs in tropical Africa and the islands of the Indian Ocean. This work concludes a study that describes the morphology of the species occurring in São Paulo state (Brazil), together with a key for identification. A total of six species with flat joints is recognized for São Paulo state.
\end{abstract}

Key words: Rhipsalis, taxonomy, flat joints

\section{Introdução}

O gênero Rhipsalis foi descrito por Gärtner (1788), com uma única espécie, $R$. cassytha $(=R$. baccifera (J.Miller) Stearn). O gênero, segundo Mabberley (1987), possui cerca de 50 espécies, que se distribuem pela América do Sul, África tropical, Madagascar e ilhas do Oceano Índico até Sri-Lanka, as espécies de ramos predominantemente aplanados no entanto ocorrem somente no continente americano (Barthlott, 1983).

\footnotetext{
' Parte da dissertação de Mestrado apresentada ao Departamento de Botânica da Universidade Estadual de Campinas, e financiado em parte por bolsa CAPES (Demanda Social).

2 Departamento de Botânica - ICB - UFMG - Caixa Postal 478 - 31270-110 - Belo Horizonte - MG.
} 
Dentre os principais autores que trataram do gênero destacam-se Schumann (1890) que considerou oito espécies com ramos aplanados para o Brasil, Loefgren $(1915,1918)$ que reconheceu 10 espécies no Brasil, Britton \& Rose (1923) que relacionaram 12 destas espécies brasileiras e Backeberg (1959) que também reconheceu 12 espécies de Rhipsalis com ramos aplanados. Mais recentemente Scheinvar (1985) citou sete espécies de ramos aplanados para o Estado de Santa Catarina. Para o Estado de São Paulo não existem citações específicas de quais espécies ocorrem, no entanto as referências acima relacionam cerca de oito espécies de ramos aplanados que ocorreriam no Estado.

A primeira parte deste trabalho (Lombardi, 1991) descreveu e apresentou uma chave de identificação para 16 espécies de Rhipsalis que possuem ramos cilíndricos a subcilíndricos mas nunca predominando ramos aplanados. Neste presente trabalho são apresentadas descrições sucintas, ilustrações e chave de identificação de outras seis espécies do gênero que ocorrem no Estado de São Paulo e apresentam predominantemente ramos aplanados ou foliáceos, carregando aréolas apenas nas margens dos ramos.

\section{Material e métodos}

Foram examinados materiais herborizados depositados em herbários nacionais e estrangeiros, coletados no Estado de São Paulo e em outros Estados do país, a fim de comparação. Para as descrições das características morfológicas foram usados os exemplares herborizados provenientes de todo o Brasil, mas preferencialmente os procedentes de São Paulo. Exemplares vivos foram coletados em várias localidades do Estado e mantidos em casa de vegetação, sendo usados para o exame das flores e frutos, além de permitir a verificação da permanência das características quando os indivíduos eram cultivados juntos no mesmo ambiente. Os sinônimos estão de acordo com a literatura citada e são sugeridos sinônimos prováveis dentre os nomes publicados. Nas citações de material examinado foram usados materiais do Estado de São Paulo ou, na falta destes, os de procedência mais próxima.

\section{Descrição do gênero}

Rhipsalis Gärtner, Fruct. Sem. I: 137, 1788

Hariota Adanson, Fam. Pl. 2: 243, 1763 (non DC,1834), nom. rej.

Cassyta J.Miller, Gard. Dict. ed.8, 1768 (non DC,1834)

Erythrorhipsalis Berger, Monatsschr. Kakteenk. 30: 4, 1928

Plantas epífitas ou rupícolas, pendentes. Ramos articulados (artículos ou cladódios) cilíndricos, costados, angulados, alados a aplanados ou foliáceos (às vezes combinando de duas a três formas); ramificação terminal ou lateral, com raízes adventícias ocasionais. Aréolas de zonas meristemáticas cavernosas (imersas no ramo em um alvéolo) ou emersas, distribuídas ao longo do caule e em suas extremidades, ou 
ângulos ou nas margens dos ramos aplanados, com cerdas, tricomas e mesmo espinhos diminutos. Folhas ausentes ou reduzidas a esćamas paleáceas diminutas. Flores pequenas para a família, emersas ou em alvéolo afundado no ramo, laterais ou terminais, de solitárias até várias na mesma aréola, de inodoras até de odor agradável, actinomorfas, com a corola reflexa ou campanulada, epigíneas. Hipanto ausente ou muito reduzido. Tépalas organizadas em espiral, desiguais entre si, de 6 a 25 , as mais exteriores diminutas, escamiformes, triangulares, de ápice agudo, de verdes até avermelhadas, as mais interiores de elípticas até obovadas, com ápice cuculado até agudo, em número de quatro até mais de dez. Estames de poucos até mais de cem. Filetes translúcidos, esverdeados ou brancos, às vezes avermelhados na base. Estilete cilíndrico, central, translúcido, esverdeado ou branco, às vezes avermelhado na base ou até quase a sua metade. Estigma de tri até octolobado, lobos brancos, papilosos na face interna. Disco nectarífero anular, esverdeado ou avermelhado. Ovário ínfero, imerso no pericarpelo. Pericarpelo de tecido caulinar, englobando o ovário propriamente dito e participando da formação do fruto, cônico ou elíptico, cilíndrico ou angulado, emerso ou imerso na superfície do ramo, nu ou com aréolas, escamas e até cerdas, quando maduro de branco translúcido e amarelo até púrpura. Fruto baga, com polpa sucosa e viscosa, esférico, elíptico ou até discóide. Sementes pequenas, reniformes, oblongas, ovaladas ou circulares, de cor negra brilhante até marrom, com testa lisa ou reticulada. Plântulas com caule primário com ângulos ou aplanados, e aréolas espinescentes.

\section{Chave para a identificação das espécies}

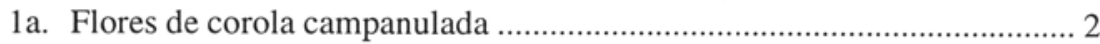

1b. Flores de corola rotácea .......................................................................... 4

2a. Ramos elípticos com base constricta e margem serreada .... I.R. houlletiana

2b. Ramos lineares ou lanceolados e margem crenulada ............................. 3

3a. Flor ca. 2,0cm compr.; ca. 1,5cm diâm.; branca a sépia .. II.R. warmingiana

3b. Flor ca. 3,5cm compr.; ca. 2,5cm diâm., branca .................... III.R. linearis

4a. Lâmina dos ramos rômbicas a oblongas, crassas; crenas pronunciadas com espinhos curtos, fruto branco IV.R. pachyptera

4b. Lâmina dos ramos elípticas ou obovadas, crenas atenuadas, sem espinhos, fruto vermelho a rosado. 5

5a. Ramos ca. 3,0cm larg., ca. 9,0 cm compr, delgados

5b. Ramos ca. 5,0cm larg., ca. 13,0cm compr., crassos V.R. rhombea VI.R. platycarpa

\section{Descrição das espécies}

I. Rhipsalis houlletiana Lem., Ill. Hort. 5: Misc. 64, 1858

$R$. regnellii Lindbg., Gartenflora 39: 119, 1889

Hariota houlletiana Kuntze, Revis. Gen. Pl. 1: 263, 1891 
Ramos elípticos a lanceolados com a base constricta na base ou até além da metade, ca. 23,0 cm compr., ca. 3,5 cm larg., lâmina dos ramos delgada, eixo central proeminente, margem profundamente serreada. Aréolas com escama triangular e pêlos curtos escassos. Flores de corola campanulada, ca. 2,0 cm compr., ca. 1,5 cm diâm. Tépalas ca. 12, as mais externas triangulares, esverdeadas com a extremidade avermelhada; as mais internas elípticas, branco-amareladas. Estames ca. 35. Estigma trilobado. Pericarpelo elíptico, tetra- a pentagonal, nu. Fruto globoso, ca. $0,8 \mathrm{~cm}$ diâm, vermelho. Sementes sub-oblongas, castanho-avermelhadas. (FIG.1a, 1b)

Distribuição. Brasil: Minas Gerais, Rio de Janeiro, São Paulo, Paraná, Santa Catarina, Rio Grande do Sul. Argentina.

Material examinado: MINAS GERAIS: Caldas, Regnell III-626, 1861 (F, S). RIO DE JANEIRO: Parque Nacional do Itatiaia: Brade 14559, 22/05/1935 (RB); Rio de Janeiro: Floresta da Tijuca, Angeli 72, 18/06/1960 (GUA); s/local., Glaziou 14861, 1885 (G). SÃO PAULO: Apiaí: Puigari s/n, 08/1885 (P); Atibaia: Bernacci et al., 02/06/1987 (UEC 55001); Bananal: Brade 15224, 21/05/1936 (RB); Botucatu: Bicudo, 21/07/1981 (BOTU 11836); Paranapiacaba: Zappi 55, 13/05/1988 (UEC); Parque Estadual de Carlos Botelho: Lombardi 131, 07/06/1992 (UEC); São Paulo: Serra da Cantareira, Aguiar \& Baitello, 30/06/1978 (SPSF 5735); São Paulo: Parque Estadual das Fontes do Ipiranga, Handro, 15/07/1941 (SP 48797); São Paulo: Horto do Museu Paulista, Hoehne, 07/07/1933 (SPF 10292); São Sebastião: Lombardi, 17/06/1989 (UEC 51470). PARANÁ: Cêrro Azul: Hatschbach 48112, 19/07/1984 (MO); Curitiba: Cordeiro \& Silva 331, 31/07/1986 (UEC); Guarapuava: Lindeman \& de Haas 4992a, 18/03/1967 (U); Ipiranga: Dusén 17257, 12/09/1915 (S); Itaperucu: Dusén 7164, 17/11/1908 (S); Jacarezinho: Dusén, 24/04/1915 (S); Morretes: Cordeiro \& Zelma 301, 10/07/1986 (HRB); São Mateus do Sul: Souza et al., 17/07/1986 (UEC 48938); Terrinha: Dusén 7334, 09/12/1908 (P, S); Vila Velha: Brade 19587, 15/02/ 1949 (RB). SANTA CATARINA: Curitibanos: Klein 3147, 18/09/1962 (UC); Itajaí: Klein 2068, 24/05/1956 (B, BR, G, UC); Lajes: Klein 2931, 13/09/1962 (BR); Papanduva: Klein 2967, 14/09/1962 (B, G). RIO GRANDE DO SUL: Colônia Santo Ângelo: Lindman 1051, 13/01/1893(S); Montenegro: Friederichs 32934, 02/10/1945 (B). SEM LOCALIDADE PRECISA: Lindberg, 1890 (S); sem coletor, s/data (MO 2287878, F 1794054, RB 7058, UC 1387624).

ARGENTINA: MISSIONES: Fracran, Hauman s/n, 23/02/1924 (BA 24831).

Esta espécie é muito ornamental, facilmente distinguível das demais espécies por seus ramos de bordas profundamente serreadas e suas flores grandes e inclinadas para o centro da lâmina do ramo. Em estado vegetativo $R$. houlletiana pode ser confundida a primeira vista com Epiphyllum phyllanthus (L.) Haw. que possui ramos mais grossos e rijos com as bordas crenadas. Barthlott (1987) transferiu tentativamente esta espécie para o gênero Lepismium, no entanto a razão desta mudança permanece 


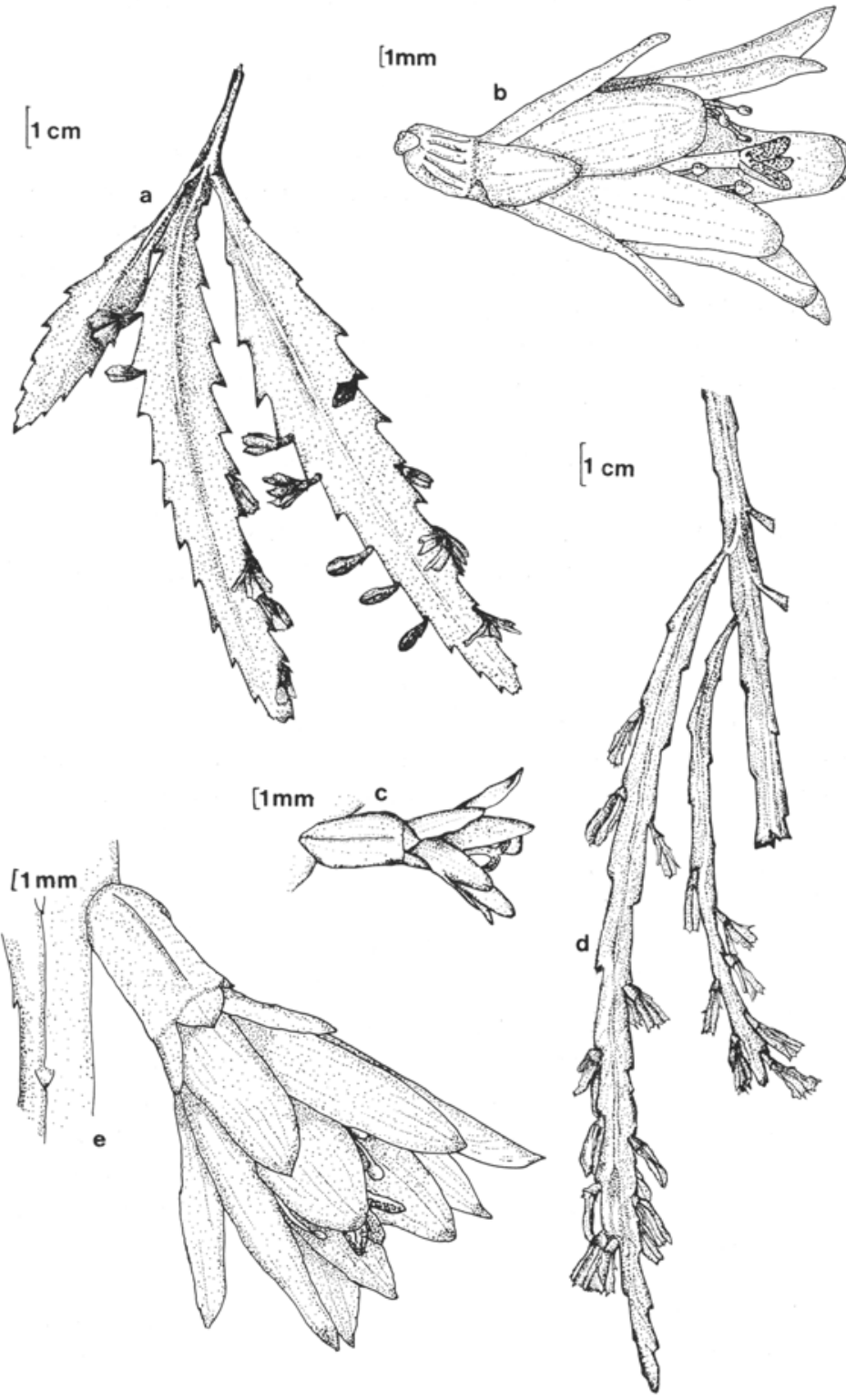

FIG. 1-Rhipsalis houlletiana Lem., a-aspecto geral do ramo, b-flor (Dusén 17257). Rhipsalis warmingiana Schum., c-flor (Lombardi 148). Rhipsalis linearis Schum., d-aspecto geral do ramo (Souza et al. 302), eflor (Lombardi 149). 
a ser explicada, possivelmente em uma revisão do gênero Rhipsalis e outros afins em preparação por Barthlott, Taylor \& Zappi (Taylor, com. pessoal).

II. Rhipsalis warmingiana Schum. in Mart., Fl. bras. 4(2): 291, 1890

Ramos lanceolados, esporadicamente tri- a tetra-alados, ca. $23,5 \mathrm{~cm}$ compr., ca. $2,0 \mathrm{~cm}$ larg., margem crenada. Aréolas com escama triangular, pêlos curtos escassos e uma ou duas cerdas curtas. Flores de corola campanulada, ca. 2,0 cm compr., ca. 1,0 cm diâm., inodoras. Tépalas 15, as mais externas triangulares, avermelhadas; as mais internas fusiforme-lanceoladas, sépia a esbranquiçadas. Estames ca. 30. Estigma tetralobado. Pericarpelo elíptico, tetra- a poli-angulado, nu ou com escama. Fruto globoso, ca. 1,0 cm diâm., púrpura a negro. Sementes subelípticas, negras. (FIG.1c)

Distribuição. Brasil: Minas Gerais, São Paulo.

Material examinado: SÃO PAULO: Campinas: Lombardi 148, 16/10/1992 (UEC); Cerqueira César: Gounelle 100, 12/1898 (G). MINAS GERAIS: Caldas: Lindberg 511, 10/1854 (syntypus: S); Caldas: Lindberg 611, 10/1854 (BR, MO).

Rhipsalis warmingiana apresenta pequenas flores de cor sépia, o que a torna facilmente distinta das outras espécies aqui tratadas, vegetativamente esta espécie assemelha-se a $R$. linearis, mas distingue-se pelas flores muito menores, de cor distinta, e pelos ramos mais largos. Barthlott (1987) também transferiu esta espécie para o gêneroLepismium, como em R. houlletiana no entanto sem explicar os critérios utilizados na sua transferência.

III. Rhipsalis linearis Schum. in Mart., Fl. bras. 4(2): 296, 1890

Ramos linear-lanceolados, esporadicamente tri a tetra angulados, ca. $24,0 \mathrm{~cm}$ compr., ca. $0,7 \mathrm{~cm}$ larg., margem crenulada. Aréolas com escama triangular. Flores de corola campanulada, ca. 3,0cm compr., ca. 2,5cm diâm., inodoras. Tépalas 11, as mais externas triangulares, branco-avermelhadas; as mais internas elípticas, branco-esverdeadas. Estames ca. 30. Estigma tetra- a pentalobado. Pericarpelo cônico, pentaangulado, com escama ou nu. Fruto elíptico, ca. $1,0 \mathrm{~cm}$ compr., ca. $0,7 \mathrm{~cm}$ diâm., púrpura. Sementes subrômbicas, castanho-escuras. (FIG.1d, 1e)

Distribuição. Brasil: São Paulo, Paraná, Santa Catarina, Rio Grande do Sul. Argentina. Paraguai.

Material examinado: SÃO PAULO: Atibaia: Lombardi 149, 1988 (UEC); Botucatu, Joamas Jr. 2, 19/09/1972 (BOTU). PARANÁ: Curitiba: Dusén 17198, $12 /$ 09/1915 (F, MO, S); Ipiranga: Dusén 8811, 22/08/1909 (P, S); Londrina: Ogido \& Ikuta, 20/08/1980 (FUEL 292); Ponta Grossa: Hatschbach 14645, 05/09/1966 (B, 
MO); Rio Branco do Sul: Klein 2494, 25/08/1961 (GUA); São Mateus do Sul: Britez et al. 900, 10/09/1986 (UEC). SANTA CATARINA: Descanso: Castellanos 24845, 02/03/1964 (GUA); Rio das Flôres: Castellanos 24802, 01/03/1964 (GUA); Rio do Sul: Lourteig 2315, 17/07/1967 (P). RIO GRANDE DO SUL: Farroupilha: Camargo 2625, 25/11/1957 (B); Porto Alegre: Czermak \& Reineck 547, 02/07/1897 (G); Rio Pardo: Juergens 436, 09/1931 (B); sem localidade, Schwabenschneib, Rambo 41778, 25/05/1949 (BR); sem localidade, Isabelle, 1835 (G 7019-31); sem localidade, Lindman, 1893 (S). SEM LOCALIDADE PRECISA: Sellow 3979 (syntypus: MO); Sellow 1869 (MO).

PARAGUAI: sem localidade, Balansa 2500 [foto do syntypus] (F).

ARGENTINA: MISSIONES: Fracran, Hauman s/n, 23/02/1924 (BA 24830).

Esta espécie apresenta grandes flores brancas muito ornamentais e ramos estreitos, distinguindo-se claramente de Rhipsalis warmingiana nestas características. $R$. gonocarpa Weber é provavelmente um sinônimo desta espécie, descrito com base em um exemplar de frutos imaturos verdes, no entanto como o tipo de $R$. gonocarpa não foi localizado este nome não foi sinonimizado aqui. Apesar da evidente proximidade de $R$. linearis com $R$. warmingiana, Barthlott (1987) não transferiu esta espécie para o gênero Lepismium.

IV. Rhipsalis pachyptera Pfeiff., Enum. Cact.: 132, 1837

Cactus alatus Willd., Enum. Pl. Suppl.: 35, 1813 (non Swartz, 1788)

Epiphyllum alatum Haw., Suppl. Pl. Succ.: 84, 1819

Cactus triqueter Vell., Fl. Flum.: 206, 1825 (non Haw. 1803 nec Willd. 1813)

Cereus alatus Link \& Otto, Icon. Pl. Rar.: 77, 1830

Lepismium fluminense Miq., Bull. Neérl.: 48, 1838

$R$. robusta Lem., Rev. Hort., 9: 502, 1860

R. pachyptera var. purpurea Cord., Gard. Chron. 2: 468, 1887

Hariota triquetra (Vell.) Kuntze, Revis. Gen. Pl. 1: 263, 1891

Hariota pachyptera (Pfeiff.) Kuntze, ibid.

Hariota robusta (Lem.) Kuntze, ibid.

$R$. crassa Schum., Keys: 54, 1903

Ramos rômbicos a oblongos, ca. 13,0 cm compr., ca. 6,5 cm larg., margem com crenas pronunciadas, ramos basais tri- ou tetra-alados. Aréolas com escama triangular, nos ramos mais velhos com 3-4 espinhos curtos. Flores de corola reflexa, ca. $1,2 \mathrm{~cm}$ compr., ca. $1,5 \mathrm{~cm}$ diâm., 1 a 5 por aréola, odor agradável. Tépalas ca. 10, as mais externas triangulares, esverdeadas com a borda avermelhada; as mais internas oblongo-lanceoladas, esverdeadas. Estames ca. 70. Estigma tetra- a hexalobado, lobos às vezes bipartidos. Pericarpelo globóide com escama avermelhada. Fruto globóide, ca. 0,6 cm diâm., branco-esverdeado a avermelhado. Sementes elipsóides, castanhoescuras. (FIG.2a, 2b) 


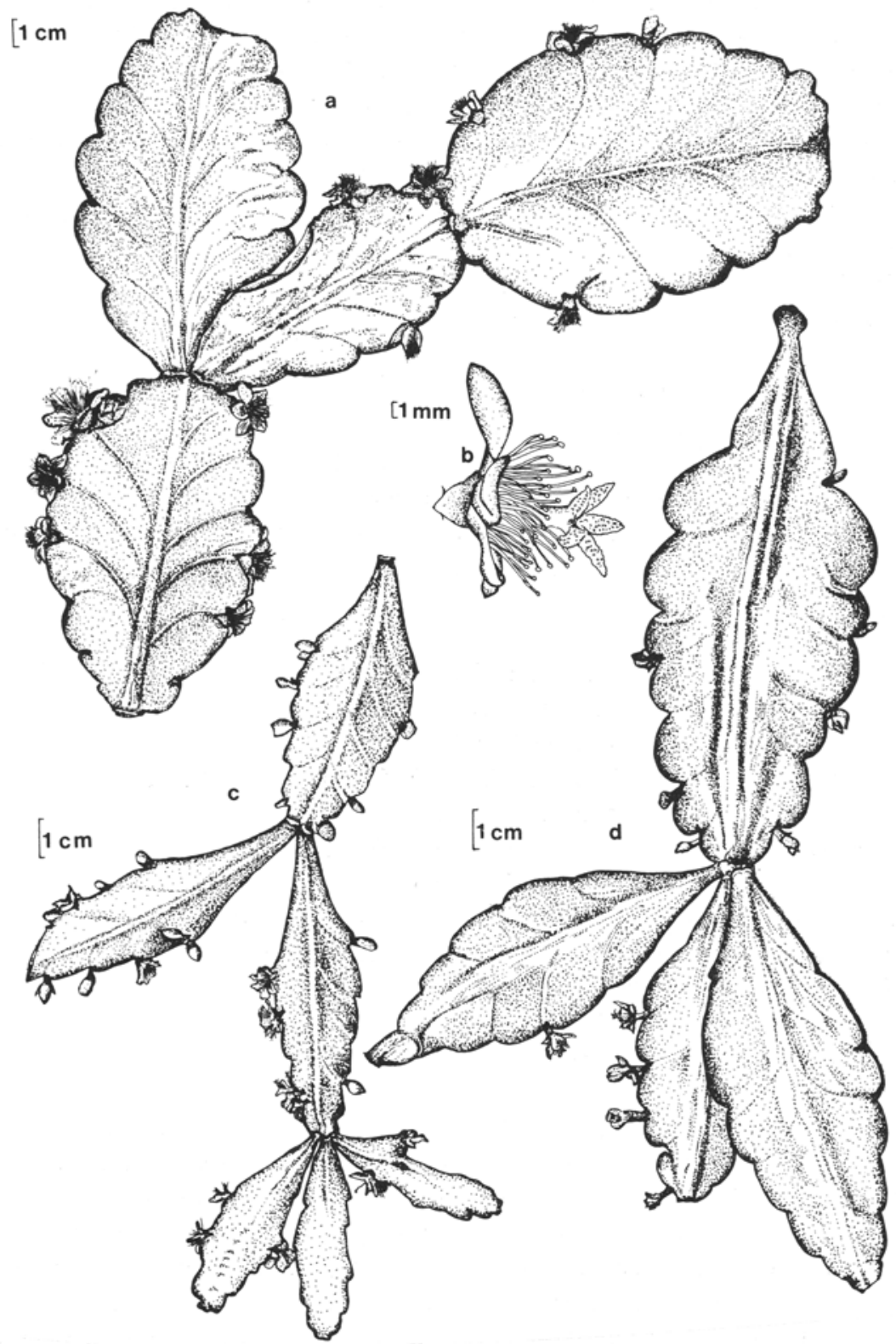

FIG. 2-Rhipsalis pachyptera Pfeiff. a-aspecto geral do ramo (s/col., UC 1387650), b-flor (Lombardi 150). Rhipsalis rhombea (S-D.) Pfeiff., c-aspecto geral do ramo (s/col., F 1794060). Rhipsalis platycarpa (Zucc) Pfeiff., d-aspecto geral do ramo (Costa \& Ribeiro 211). 
Distribuição. Brasil: Rio de Janeiro, São Paulo, Paraná, Santa Catarina.

Material examinado: RIO DE JANEIRO: Angra dos Reis: Ilha Grande, Araújo et al. 6214, 11/04/1984 (GUA); Mangaratiba: Castellanos 24016, 20/01/1962 (GUA); Parati: Martinelli 554, 10/01/1974 (RB); Petrópolis: Glaziou 14862, 09/06/1883 (P); Rio de Janeiro: Recreio dos Bandeirantes, Duarte \& Pereira 4807, 12/05/1959 (RB); Vassouras, Nagelschmidt, 09/06/1960 (F). SÃO PAULO: Cananéia: Pirani \& Yano 538, 24/1 1/1983 (SPF); São Paulo: Parque Estadual das Fontes do Ipiranga, Hoehne, 07/05/1954 (SPF 15349); São Paulo: Loefgren, 1884 (S); Ubatuba: Lombardi 150, 1986 (UEC). PARANÁ: Antonina: Hatschbach 46275, 28/04/1983 (MO). SANTA CATARINA: Brusque: Smith 5812, 23/02/1952 (RB). SEM LOCALIDADE PRECISA: sem coletor, (F 1793283, MO 2287790, P, UC 1387650, UC 1387651).

Espécie muito frequente na mata Atlântica, distingue-se de Rhipsalis rhombea $\mathrm{e}$ de $R$. platycarpa pela forma e tamanho dos ramos, pela presença de espinhos nas aréolas e pela cor do fruto. A validade das espécies $R$. crispata (Haw.) Pfeiff. e $R$. crispimarginata Loefgren em relação a $R$. pachyptera, $R$. rhombea e $R$. platycarpa permanece a ser verificada, tratando-se provavelmente de simples variantes ambientais com a margem dos ramos crispada.

V. Rhipsalis rhombea (S-D.) Pfeiff., Enum. Cact.: 130, 1837

Cereus rhombeus S.-D., Cact. Hort. Dyck.: 341, 1834

Hariota rhombea Lem., Cact. Gen. Nov. Sp.: 75, 1839

Ramos elípticos a ovalados, ca. 9,0 cm compr., ca. 3,0 cm larg., margem crenulada, ramos basais alados. Aréolas com escama triangular e uma ou duas cerdas curtas. Flores de corola reflexa, ca. 1,0 cm compr., ca. $1,1 \mathrm{~cm}$ diâm., 1 a 4 por aréola. Tépalas ca. 8, as mais internas elípticas. Estames ca. 85. Estigma tetralobado. Pericarpelo globoso, nu ou com escama. Fruto subglobóide, ca. 0,5 cm diâm., vermelho. Sementes elípticas, negras. (FIG.2c)

Distribuição. Brasil: Rio de Janeiro, São Paulo, Paraná, Santa Catarina.

Material examinado: RIO DE JANEIRO: Angra dos Reis: Pedrosa 1049, 15/02/ 1984 (GUA); Cabo Frio: Sucre 3955, 14/10/1958 (MO). :Rio de Janeiro: Floresta da Tijuca, Glaziou 14859, 10/07/1883 (P); Terezopólis: Parque Nacional da Serra dos Orgãos, Maas \& Martinelli 3397 (UEC); s/local., Gaudichaud 914, 1834 (G). SÃO PAULO: Botucatu: Hernandes Bicudo, 15/03/1982 (BOTU 11835); São Paulo: Parque Estadual das Fontes do Ipiranga, Silvestre 231, 19/03/1980 (SP); Ubatuba: Lombardi 152, 12/1990 (UEC). PARANÁ: Campina Grande do Sul: Hatschbach 9056, 18/02/1962 (RFA).SANTA CATARINA: Vidal Ramos: Reitz \& Klein 4369, 15/06/1957 (BR). BRASIL: s/local., Rose (UC 13877656); s/local., Rose 14112, 10/ 
10/1940 (UC); s/local., Rose, 10/10/1940 (F 1792936, F 1794060, MO 2287902); s/ local., s/coletor (F 1792948, MO 2287785, P, UC 1387613, UC 1387615, UC 1387618, UC 1387661).

$R$. rhombea distingue-se de $R$. platycarpa basicamente por possuir os ramos nitidamente menores e delgados, caracter que é mantido mesmo quando as duas espécies são mantidas em cultivo sob as mesmas condições em casas de vegetação.

VI. Rhipsalis platycarpa (Zucc.) Pfeiff., Enum. Cact.: 131, 1837

Epiphyllum platycarpum Zucc., Cat. Cact. Monac., 1836

Cereus platycarpus (Zucc.) Zucc., Abh. Bayer. Akad. Wiss., Math.-Naturwiss. Kl. 1: 263, 1891

Hariota platycarpa (Zucc.) Kuntz., Revis. Gen. Pl. 1: 263, 1837

Ramos elípticos a ovados, ca. 12,5 cm compr., ca. 4,5 cm larg, margem crenada, ramos basais alados. Aréolas com escama triangular. Flores de corola reflexa, ca. 0,9 cm compr., ca. 1,5 cm diâm., 1-2 por aréola. Tépalas ca. 9, as mais externas triangulares, esverdeadas; as mais internas elípticas, brancas. Estames ca. 80. Estigma tetralobado. Pericarpelo fusiforme, nu ou com escama. Fruto globóide, ca. $0,5 \mathrm{~cm}$ diâm., vermelho a rosado. Sementes fusiformes, pretas. (FIG.2d)

Distribuição: Rio de Janeiro, São Paulo, Paraná, Santa Catarina.

Material examinado: RIO DE JANEIRO: Angra dos Reis: Araújo \& Maciel 3997, 17/09/1980 (GUA); Angra dos Reis: Ilha Grande, Araújo et al. 5710, 27/09/ 1983 (GUA); Itatiaia: Barreto 121, 13/02/1980 (HRB); Jacarépagua: Vilaça 92, 29/ 10/1980 (GUA); Petrópolis: Ribeiro 698, 21/09/1985 (GUA); Rio de Janeiro: Grota Funda, Castellanos 23146, 05/1962 (GUA); Rio de Janeiro: Parque Estadual Pedra Branca, Costa \& Ribeiro 211, 28/08/1982 (GUA); Rio de Janeiro: vertente NW da Serra da Piaba, Sucre 7052, 17/08/1970 (GUA, UEC); Rio de Janeiro: Floresta da Tijuca, Strang 166, 18/05/1960 (GUA); Teresópolis: Parque Nacional da Serra dos Órgãos, Castellanos 23172, 24/10/1961 (GUA). SÃO PAULO: Bananal: Martinelli 4672, 23/06/1978 (UEC); Campinas: Taroda et al. 18601, 30/04/1986 (UEC); Cunha: Kiehl \& Franco s/n 18/10/1939 (IAC); Santo André: Rosa \& Pires 3975, 27/11/1980 (MG); São Paulo: Parque Estadual das Fontes do Ipiranga, Kirizawa 546, 07/05/1980 (SP). PARANÁ: Campina Grande do Sul: Hatschbach 9105, 01/04/1962 (B); Guaraqueçaba: Hatschbach 49349, 08/05/1985 (G, HRB, MG, UEC); Paranaguá: Ilha do Mel, Silva \& Souza s/n, 26/11/1987 (UEC 53958). SANTA CATARINA: Luís Alves: Reitz \& Klein 2888, 22/03/1956 (BR, G, UC); Palhoça: Smith 6223, 14/03/1952 (RB); Vidal Ramos: Reitz \& Klein 6658, 08/04/1958 (B, G, UC). SEM LOCALIDADE PRECISA: Sucre 10957, s/data (UEC); Lindberg, s/data (S); sem coletor, 1918 (RB 8835). 
R. platycarpa e R. rhombea, são distinguíveis basicamente pelo tamanho dos ramos, característica que é mantida sob cultivo das espécies nas mesmas condições, talvez estes dois taxa devessem ser considerados como subespécies de $R$. rhombea. $R$. platycarpa foi descrita como possuindo fruto verde com bordas avermelhadas, provavelmente um fruto imaturo, hipótese já levantada por Britton \& Rose (1923). $R$. elliptica é provavelmente um sinônimo de $R$. platycarpa, sendo distinguidas unicamente pela cor do fruto.

\section{Agradecimentos}

O autor agradece à Profa. Dra. Graziela Maciel Barroso pela orientação na dissertação; ao Prof. João Semir pelas sugestões e esclarecimentos; a Celina Mitiko Yokoro pela revisão ortográfica; ao Prof. George John Shepherd pela elaboração do Abstract; aos Drs. Urs Eggli, Wilhem Barthlott, Beat Leuenberger, Roberto Kiesling, S.A. Volgin, Mr. P.V. Heath e Myron Kimnach pelo gentil envio de cópias e separatas; e aos curadores dos herbários B, BA, BR, BOTU, F, FUEL, G, GUA, HRB, IAC, MG, MO, P, RB, RFA, S, SP, SPF, SPSF, U, UC, UEC e UPCB, pelo envio do material examinado.

\section{Referências bibliográficas}

Backeberg, C. 1959. Die Cactaceae-Handbuch der Kakteenkunde II, Gustav Fischer Verlag, Jena.

Barthlott, W. 1983. Biogeography and evolution in Neo- and Paleotropical Rhipsalinae (Cactaceae), Sondberb. Naturwiss. Vereins Hamburg 7: 241-248.

Barthlott, W. 1987. New names in Rhipsalidinae (Cactaceae), Bradleya 5: 97-100.

Britton, N.L. \& J.N. Rose. 1923. The Cactaceae. Descriptions and Illustrations of Plants of the "Cactus" Family IV, Carnegie Institution, Washington.

Gärtner, J. 1788. De Fructibus et Seminibus Plantarum I, Academiae carolinae, Stuttgart.

Loefgren, A. 1915. O gênero Rhipsalis, Arch. Jar. Bot. Rio de Janeiro 1: 59-104.

Loefgren, A. 1918. Novas Contribuições para o gênero Rhipsalis, Arch. Jar. Bot. Rio de Janeiro 2: 34-45.

Lombardi, J.A. 1991. O gênero Rhipsalis Gaertner (Cactaceae), no Estado de São Paulo. I.Espécies com ramos cilíndricos ou subcilíndricos. Acta bot. bras. 5(2): 53-76.

Mabberley, D.J. 1987. The Plant-Book, Cambridge University Press.

Scheinvar, L. 1985. Cactáceas. In: Reitz, R. (ed.) Flora Ilustrada Catarinense.

Schumann, K.M. 1890. Cactaceae. In: Martius, C.F.P. (ed.) Flora Brasiliensis 4(2): 266-300. 